\title{
Generalized Potentials Describing Orbits in the Solar System: Derivation of a 'Close-Force' Acting on the Inner Moon Phobos
}

\author{
Lena Strömberg \\ Previously Department of Solid Mechanics, Royal Institute of Technology, KTH (Kungl Tekniska Hogskolan), Stockholm 11800, Sweden
}

Received: October, 11, 2014 / Accepted: December, 30, 2014 / Published: January, 30, 2015

\begin{abstract}
The increased velocity for the inner moon Phobos at Mars is calculated, assuming a modified gravity law with distributed mass. The motion of the moon is assumed to be balanced by the "close-force". The results from Strömberg, for planets in noncircular orbits, in conjunction with assuming a density, admit a balance equation for the rotation in a continuum flow. From this, explicit expressions for a velocity field and a so called Le-density are given. These are exploited to model distributed mass and formations, exemplified with a large asteroid, 2 Pallas, in the asteroid belt, bounds for the L-frequency, the formation of Mercury and distances between planets.
\end{abstract}

Key words: Celestial mechanics, phobos, non-newtonian, close-force, distributed rotating mass, rotational field, Le-density field.

\section{Introduction}

Most of the planets and moons move in orbits fulfilling classical Newtonian gravity with potential $\mathrm{GMm} / \mathrm{r}$, where $\mathrm{G}$ is the universal gravity constant, $\mathrm{M}$ is the mass of the primary, $\mathrm{m}$ is the mass of the planet and $r$ is the distance between the planet and primary. In the present context, some refined models will be considered.

A generalized gravity law, similar to Chandrasekhar-type [1], is assumed to calculate the increased velocity for the inner moon Phobos, Fig. 1, at Mars.

A relation between the density and angular velocity will be derived for Earth.

For planets in noncircular orbits, results from Ref. [2] will be exploited, to model distributed mass and formations. With an extension to continuum mechanics, field properties will be considered in an eccentricity zone, and a Le-density is calculated. This is applied to describe asteroid 2 Pallas, considered as a

Corresponding author: Lena Strömberg, M.Sc., research field: nonlinear mechanics. E-mail: lena_str@hotmail.com. part of a continuous mass distribution consisting of asteroids. The configuration also provides bounds for the L-frequency from Ref. [2]. Conditions and symmetry constraints for the velocity field will be considered to deduce a possible origin for the formation and location of Mercury.

\section{Central Force at Non-homogeneous Mass Distribution-Close-Force}

At locations in vicinity of a planet with non-homogeneous mass-distribution, e.g., for moons inside synchronous limit, the classical Newtonian force is not applicable. A more accurate description is obtained when the moon is considered part of the primary planet. Here, a weak form of the gravity law will be considered and the resulting equation is somewhat similar to the descriptions in Chandrasekhar [1]. This gives the orbital velocity of moons or other object, close to the primary planet.

\subsection{Integral Formulation with Density Distribution}

Consider a sphere with center at Mars, and with 
radius to Phobos. Assume that Newtonian gravity is replaced with a weak formulation, which is an integral over this volume; $f\left(\left(G \rho(r) / r^{2}\right) d V\right)$, where $\rho$ is the density distribution for Mars and the moon, which will be specified. Mean value theorem, is applied for the entire part except volume element. Hereby the expression reads $\left(G \rho\left(r_{\eta}\right) / r_{\eta}{ }^{2}\right) V_{\text {tot }}$, where $r_{\eta}$ is a location in the interval $\left[0, r_{M}\right], r_{M}$ is the radius of Mars and $V_{t o t}$ embraces the entire configuration.

Applying Newtonian dynamics, this is assumed to balance (the specific) centripetal acceleration of Phobos.

Remark: It is possible that the motion is implied by a 1:1-coupling with sidereal rotation, and entirely ruled by this and not related to a force. The sidereal rotation is probably Newtonian.

Definition: The expression $\left(G \rho\left(r_{\eta}\right) / r_{\eta}^{2}\right) V_{\text {tot }}$ will be known as the close-force (or kinematic synchronous force) valid for close satellites.

For "normal" density distributions, this is increased compared with the classical gravity force.

Proposition: There exist configurations and density distributions, such that the angular velocity of the moon Phobos is given by $\omega_{N} R / r_{M}$, where $\omega_{N}$ is the Newtonian angular velocity (predicted by Newtonian gravity), $R$ is the distance to Phobos, and $r_{M}$ is the radius of Mars.

Proof: In brief: Let $r_{\eta}$ be the point in the interval $\left[0, r_{M}\right]$ such that $\rho\left(r_{\eta}\right) V_{M}=\rho_{\text {mean }} V_{\text {tot }}$, where $V_{M}$ denotes the volume of Mars, $\rho(r)$ is the density of the nonhomogeneous mass distribution and $\rho_{\text {mean }}$ is the mean density of entire distribution including the empty space between Mars and Phobos. The result is given with some additional simplifications, and assuming that $\rho$ is constant for Mars, compare Fig. 2.

Comparison with data for the inner moon of Mars gives agreement, to some extent: The radius of Mars is $3,376 \mathrm{~km}$, orbital radius of Phobos is $9,376 \mathrm{~km}$, and the orbital revolution times are $0.31 \mathrm{~d}$, and $1.0025 \mathrm{~d}$.

A better approximation is obtained when assuming that Mars is not homogeneous, such that the density is decreased outwards. Then, results could be obtained

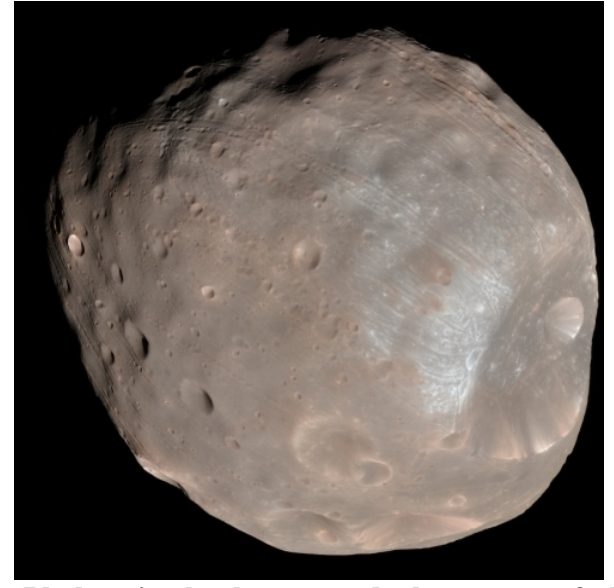

Fig. 1 Phobos is the larger and closer one of the two natural satellites of Mars. Both moons were discovered in 1877. Phobos has a mean radius of only $11 \mathrm{~km}$, and the density is $1,900 \mathrm{~kg} / \mathrm{m}^{3}$, which is less massive than Mars's $3,900 \mathrm{~kg} / \mathrm{m}^{3}$.

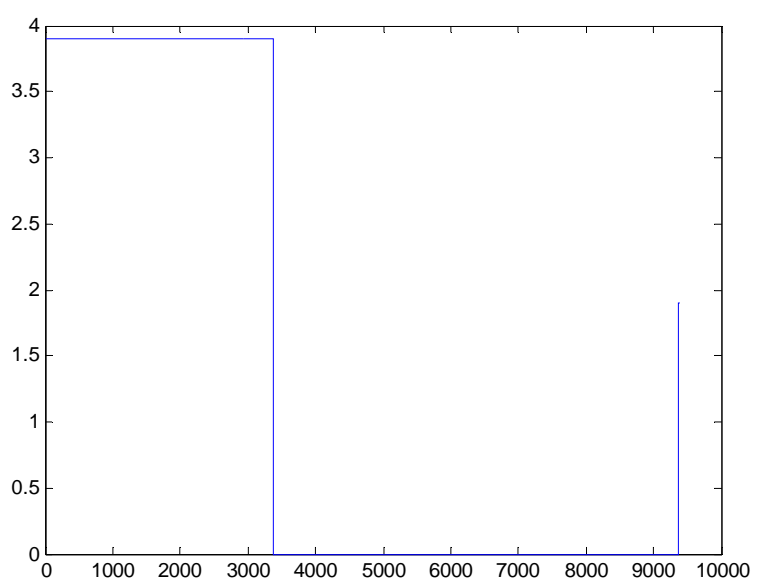

Fig. 2 Density distribution for Mars (simplified) and the moon Phobos, at 9,376 km.

by numerical evaluation of the integral, e.g., Gaussian integration.

\subsection{Example. Gas Giants}

It is possible that rocky objects approached the planet, as moons with increased velocity (inside the synchronous limit, and then, eventually passing the Roche limit and being split into a formation of planetary rings, which rotates governed by Newtonian gravity.

Remark: A weak formulation is less deterministic, however beneficial, since:

- Functions need not be continuous;

- A weight function can be invoked (as in e.g., the 
FEM (finite element method) [3])

- The integral can be evaluated approximately in different manners, in order to capture the desired correct solution.

\section{Density as Potential for Rotational Flow}

For objects in vicinity of the equator at Earth, the orbital angular velocity is proportional to the square root of density, $\omega^{2}=\rho G 4 \pi / 3$. For the other rocky planets, this relation is not valid.

\subsection{Balance of Mass}

In continuum mechanics, when no explicit dependency on time, an equation for balance of mass reads:

$$
\operatorname{div}(\rho \boldsymbol{u})=0
$$

where, $\rho$ is density and $\boldsymbol{u}$ is the velocity of the continuum. Based on this, a density will be calculated, assuming that the velocity in the eccentricity zone is a field. To obtain this continuum extension, a change of variable from $t$ to a spatial dependency is defined.

From Ref. [2], the time dependent part of radius vector creating the eccentricity zone is given by:

$$
r_{\varepsilon}=\left(r_{e c c} / r_{0}\right) \sin \left(f \omega_{0} t\right)
$$

where, $f \omega_{0}$ is the L-frequency of a noncircular orbit, and $r_{0}$ is the radius of the circle.

Definition: A decomposition is considered such that $\phi-\phi_{0}=\omega_{0} t-\omega_{\varepsilon} t$, where $\phi$ is the angle along the path at radius $r_{0}, \phi_{0}$ is a constant initial angle, $\omega_{\varepsilon}$ is a (small) angular velocity, depending on the length of the interval considered, and the eccentricity. To consider radial dependency, the angle $\phi$ is replaced with $s / r, s$ being a constant path on the circle with radius $r=r_{0}$.

Assuming on $\phi$, either tangential dependency or radial dependency, the balance equation will be analysed.

\subsection{Tangential Dependency, Le-density Field}

At tangential dependency, the velocity in tangential direction gives contribution to $\operatorname{div}(\boldsymbol{u})$. It is assumed that $\boldsymbol{u}=\omega(\phi) r(\phi) \boldsymbol{e}$, where an expression for $\omega$ is assumed as $\omega(t)=\omega_{0} \exp \left(-2\left(r_{e c c} / r_{0}\right) \sin \left(f \omega_{0} t\right)\right)$, which is consistent with the results in Ref. [2].

Proposition: With the above preliminaries $\operatorname{div}(\boldsymbol{u})=$ $\omega^{\prime}+(\omega / r) r^{\prime}$, where ' denotes differentiation on $\phi$. Then $\operatorname{div}(\boldsymbol{u})=-\left(r_{e c c} / r_{0}\right) \cos \left(f \omega_{0} t\right) f \omega(t)$. A linearisation gives $\operatorname{div}(\boldsymbol{u})=-f \omega_{0}\left(r_{e c c} / r_{0}\right) \cos \left(f \omega_{0} t\right)$.

Proof: To obtain $\operatorname{div}(\boldsymbol{u}), \omega_{0} t$ is replaced with $\phi$ through the decomposition.

Hereby $\operatorname{div}(\boldsymbol{u})=(-2+1)\left(r_{e c} / r_{0}\right) \cos \left(f \omega_{0} t\right) f \omega(t)$.

Proposition: With a linearisation of $\boldsymbol{u}$ and no source term, the density is given by:

$$
\rho=\rho_{0} \exp \left(2\left(r_{e c c} / r_{0}\right) \sin \left(f \omega_{0} t\right)\right)
$$

where, $\rho_{0}$ is a constant. This will be known as the Le-density of an eccentricity zone.

Proof: With the linearisation $\boldsymbol{u}=\omega(\phi) r_{0} \boldsymbol{e}$ and $c=0$, $\rho$ is given by a solution to Eq. (1).

Remark: Assuming that $c$ is nonzero and other decompositions, alternate expressions can be derived.

Example, Asteroids between Mars and Jupiter: The largest asteroid with eccentricity, 2Pallas, possesses $7 \%$ of all mass. Since this is a large amount of mass, the density distribution reflects this when there is one maximum on the "circle", such that $1<f<2$. To obtain values $f<2$, a generalisation of the format in Ref. [2] is required. This could be done by invoking additional configurational forces, which is interpreted to emanate from a material memory, or a coupling to sidereal rotation.

\subsection{Radial Dependency}

For a radial dependency $\operatorname{div}(\boldsymbol{u})$ will depend on the angle $\phi$ and trigonometric functions.

\subsection{Locations of Planets}

From coupling sidereal-orbital rotation $(3 / 2)[4,5]$, and ratio of angular velocities between adjacent rocky planets (also Sun and Mercury; Section 3.2), and comparing Table 1, a guess is that the factor $f$, for Mercury is around 1.6. Assuming exactly $f=\pi / 2$, and linearising such that $\phi=\omega_{0} t$, the density will have maximum at $\phi=-1$. At this location $\operatorname{div}(u)=0$, which 
Table 1 Ratio between angular velocities.

\begin{tabular}{llllllllll}
\hline Planet & Mercury & Venus & Earth & Mars & Asteroids & Jupiter & Saturn & Uranus & Neptun \\
\hline Ratio $w_{\text {inner }} / w_{\text {outer }}$ & $3.2(\mathrm{ecc})$ & 2.5 & 1.6 & 1.9 & 2.4 & 2.6 & 2.5 & 2.8 & 1.9 \\
\hline
\end{tabular}

may be considered a local rigid body rotation. Therefore at a forming process, at this location, a planet is "likely to be present". The same result is obtained with $f=3 / 2$, and $\phi=-\pi / 3$. This value of angle corresponds to a symmetry. Next, spatial extensions are proposed, to obtain other locations.

Definition: Isotropic extension. At $\phi=-1, s=-R$ at the same path. In an isotropic extension, it is assumed that the location is in the radial direction, the distance $R$, from the circular path.

Definition: Crystal extension. At $\phi=-1, s=-R$ at the same path. In a crystal extension, the new location forms a crystal in the radial direction, at the distance approximately $\operatorname{sqrt}(3) / 2 R$, from the circular path.

Remark: The predicted location agrees approximately with the distance from Mercury to Venus. The distance from Sun to Mercury is $0.4 \mathrm{au}$, differing 0.1 au due to eccentricity, and from Sun to Venus $0.7 \mathrm{au}$.

\section{Conclusions}

A generalised gravity law was used to model the motion for the inner moon of Mars.

The Newtonian gravity law for a particle mass, was replaced by a weak formulation, which admits a more detailed description with density distribution. Since the moon is so close, it is considered part of the planet, such that a density distribution for Mars and the moon interacts to give the gravity potential. An explicit expression for a generalised increased force, was derived and denoted close-force.

It is possible that the motion is ruled by the sidereal rotation, and not governed by a force, however, here it was assumed that an increased gravity force acts, close to the primary but outside Roche limit.

The result was obtained by considering mass as distributed to a generalised density. The concept is used in continuum mechanics, to simulate e.g., winds and whirls, and could also be useful in calculations for distant galaxies and clusters of stars, as an alternative or complement to introducing dark matter.

The condition is of interest, e.g., at operating [6] a Space Craft meant to launch at the planet. It is possible that the gravity field varies due to this in combination with the eccentric orbit.

Considering the stability and evolution of the Solar System, it is possible that Mars will achieve a less eccentric orbit, when the moon collapses, which, in turn, may cause a redistribution and migration of the neighbouring planets. However, this is in a distant future, scheduled to happen 30 million years from now, according to Wikipedia.

A balance equation for the rotation in a continuum flow was derived, and analysed. The velocity was assumed as a harmonic oscillator [2]. It was found that the flow is related to a Le-density field which varies on the orbit. These results were compared with a density distribution in the asteroid belt and other features considering the formation of Mercury.

Future issues: With an eccentricity zone from Ref. [2], assumed as a continuum, several results could be derived, e.g., explicit expressions for field properties. A prospect is to further justify the modeling qualitatively and quantitatively. Then, the bounds and exact value for the L-frequency may be used in other applications.

Recent modeling (observations) for the development of a Solar System in Milky Way, is a disc with rings of matter, where planets are created. The Le-density field may serve as a description of the birth of a planet in a belt of smaller objects, with mean density $\rho_{0}$. Adopting the results from the present paper, the planet will achieve an eccentric orbit, which admits an escape to a circular orbit at a neighboring location. Related topics can be found for the asteroids between Mars and Jupiter, such that there are 
continuum properties, and a density. In this context, comparison with the gaps and moons in the planetary rings of e.g., Saturn, may also be valid. These formations are discussed and modeled in Refs. [7, 8] assuming a pressure and magnetic fields.

\section{Acknowledgements}

To the author's supervisor for valuable help on the subject, to Knut och Alice Wallenberg foundation for granting earlier work, to Prof Uhlhorn, JH Argyris, B Broberg, Dr Pål Bergan, Oslo, Dr JH Walter CERN, Prof T JR Hughes, Martin and Thomas, for valuable discussion on related topics.

\section{References}

[1] Chandrasekhar. 1960. "The Virial Theorem in Hydromagnetics." Journal of Mathematical Analysis and
Applications 1 (2): 240-52.

[2] Strömberg, L. 2014. "A Model for Non-circular Orbits Derived from a Two-step Linearisation of the Kepler Laws." Journal of Physics and Astronomy Research 1 (2): 13-5

[3] Zienkiewicz, O., Taylor, R. 2005. Finite Element Method. Elsevier Science.

[4] Correia, A., and Laskar, J. 2011. "Mercury's Capture into the 3/2 Spin-Orbit Resonance Including the Effect of Core-mantle Friction." Icarus 201: 1-11.

[5] Munroe, A. 2011. The Moons of Jupiter. Knopf Doubleday Publishing Group.

[6] Wiesel, W. E. 2010. Spaceflight Dynamics. Aphelion Press.

[7] Alfven, H. 1983. "Origin, Evolution and Present Structure of the Asteroid Region.” Accessed January 23, 2015. http://en.wikipedia.org/wiki/Hannes_Alfv\%C3\% A9n.

[8] Alfven, H. 1983. "Solar System History as Recorded in the Saturnian Ring System.” Accessed January 23, 2015. http://en.wikipedia.org/wiki/Hannes_Alfv\%C3\%A9n. 